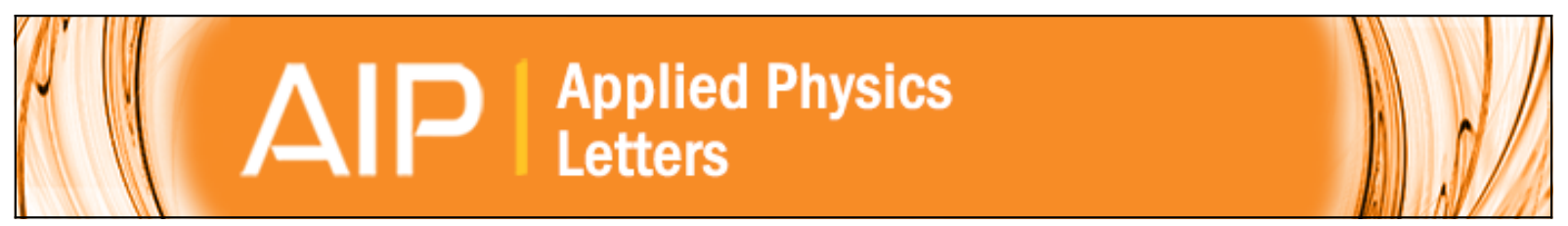

\title{
Infrared luminescence for real time ionizing radiation detection
}

Ivan Veronese, Cristina De Mattia, Mauro Fasoli, Norberto Chiodini, Eleonora Mones, Marie Claire Cantone, and Anna Vedda

Citation: Applied Physics Letters 105, 061103 (2014); doi: 10.1063/1.4892880

View online: http://dx.doi.org/10.1063/1.4892880

View Table of Contents: http://scitation.aip.org/content/aip/journal/apl/105/6?ver=pdfcov

Published by the AIP Publishing

\section{Articles you may be interested in}

Controlling energy transfer processes and engineering luminescence efficiencies with low dimensional doping J. Appl. Phys. 111, 073524 (2012); 10.1063/1.3702450

Near-infrared luminescent and antireflective in SiO2/YVO4:Yb3+ bilayer films for c-Si solar cells Appl. Phys. Lett. 99, 121110 (2011); 10.1063/1.3630003

An investigation of the thermal sensitivity and stability of the $\beta$-NaYF $4: \mathrm{Yb}$, Er upconversion nanophosphors J. Appl. Phys. 107, 054901 (2010); 10.1063/1.3298905

Conversion of infrared radiation into visible emission in YVO 4 crystals doped with ytterbium and holmium J. Appl. Phys. 96, 6323 (2004); 10.1063/1.1809270

Ytterbium-based fluorescence decay time fiber optic temperature sensor systems Rev. Sci. Instrum. 69, 4179 (1998); 10.1063/1.1149267

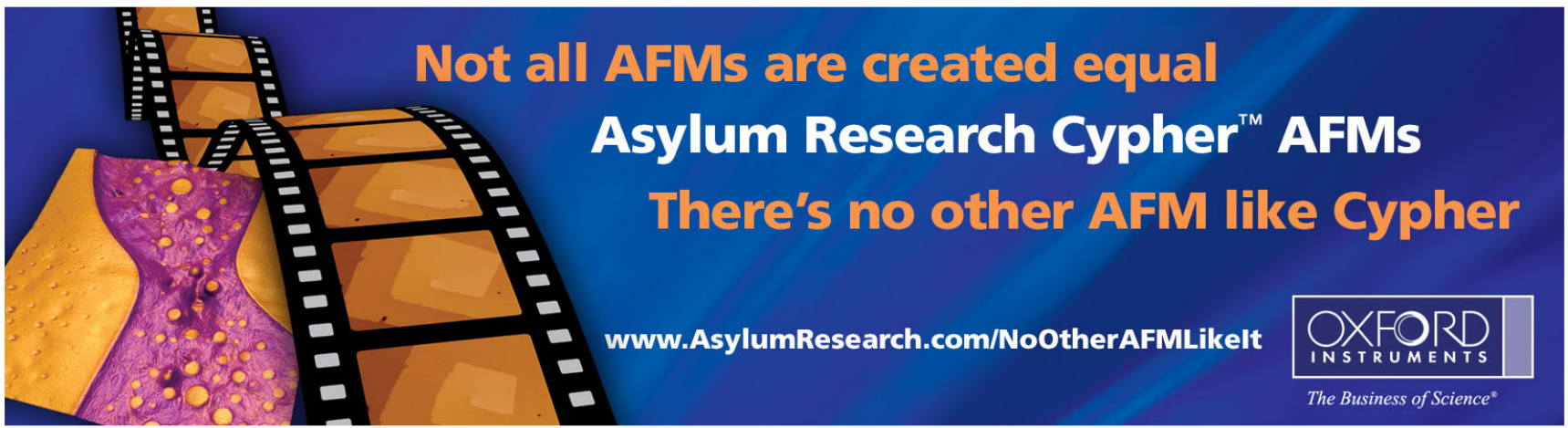




\title{
Infrared luminescence for real time ionizing radiation detection
}

\author{
Ivan Veronese, ${ }^{1, a)}$ Cristina De Mattia, ${ }^{1}$ Mauro Fasoli, ${ }^{2}$ Norberto Chiodini, ${ }^{2}$ Eleonora Mones, ${ }^{3}$ \\ Marie Claire Cantone, ${ }^{1}$ and Anna Vedda ${ }^{2}$ \\ ${ }^{1}$ Dipartimento di Fisica, Università degli Studi di Milano, Via Celoria 16, 20133 Milano, Italy \\ ${ }^{2}$ Dipartimento di Scienza dei Materiali, Università degli Studi di Milano-Bicocca, Via Cozzi 55, \\ 20125 Milano, Italy \\ ${ }^{3}$ Medical Physics Department, Azienda Ospedaliera Maggiore della Carità, Corso Mazzini 18, \\ 28100 Novara, Italy
}

(Received 7 July 2014; accepted 29 July 2014; published online 11 August 2014)

\begin{abstract}
Radio-luminescence (RL) optical fiber sensors enable a remote, punctual, and real time detection of ionizing radiation. However, the employment of such systems for monitoring extended radiation fields with energies above the Cerenkov threshold is still challenging, since a spurious luminescence, namely, the "stem effect," is also generated in the passive fiber portion exposed to radiation. Here, we present experimental measurements on Yb-doped silica optical fibers irradiated with photon fields of different energies and sizes. The results demonstrate that the RL of $\mathrm{Yb}^{3+}$, displaying a sharp emission line at about $975 \mathrm{~nm}$, is free from any spectral superposition with the spurious luminescence. This aspect, in addition with the suitable linearity, reproducibility, and sensitivity properties of the $\mathrm{Yb}$-doped fibers, paves the way to their use in applications where an efficient stem effect removal is required. (C 2014 AIP Publishing LLC.
\end{abstract}

[http://dx.doi.org/10.1063/1.4892880]

Luminescent rare-earth ions (RE) can be incorporated in silica up to considerable concentrations, despite their different ionic radius and different valence with respect to silicon. Active fiber devices could therefore be developed, like erbium doped fiber amplifiers ${ }^{1}$ and ytterbium doped laser fibers. ${ }^{2,3}$ In recent years, a further application for luminescent RE doped fibers was proposed, consisting in their employment as sensors of ionizing radiation fields. Such application, devoted for the moment mostly to medical dosimetry, exploits the phenomenon of radio-luminescence (RL), i.e., the prompt luminescence generated by ionizing radiation absorbed by the material. ${ }^{4}$ The advantage of $\mathrm{RL}$ fiber sensors with respect to other optically based sensors like those exploiting optical absorption, or optically stimulated luminescence as well as thermally stimulated luminescence is the concurrence of different qualities which are hardly found together in the other mentioned devices. We refer to the possibility to obtain a real time dose radiation field monitoring (on-line dosimetry) in a precise point (punctual evaluation), even very distant from the radiation field (remote monitoring). In practice, the dosimeter consists in a short (typically $5-15 \mathrm{~mm}$ ) portion of luminescent RE-doped silica fiber fusion spliced to a passive fiber long as desired for the transport of the emitted light to a optical detector.

The first silica based RL sensors were fabricated using cerium doping, which was selected due to the high luminescence efficiency of its $5 d_{1}-4 f$ emission centered at about $450 \mathrm{~nm} .{ }^{4-6}$ Such Ce-doped fibers were successfully applied for monitoring low energy diagnostic beams and small radiation fields. ${ }^{7,8}$ However, the measurements are problematic in the case of extended radiation fields and for beam energies above the Cerenkov threshold (approximately $190 \mathrm{keV}$ for

\footnotetext{
${ }^{\text {a) }}$ Author to whom correspondence should be addressed. Electronic mail: ivan.veronese@unimi.it
}

silica) like those employed in medical radiation therapy. ${ }^{9,10}$ In fact, in these cases the luminescence is also generated in the long passive fiber portion exposed to radiation, due to Cerenkov emission as well as to emissions of silica matrix point defects. Such signals, usually grouped together under the definition "stem effect," can be intense and hardly distinguishable from the dosimetric luminescence of the small active fiber portion due to their significant spectral superposition in the near ultraviolet-visible (UV/VIS) region. Some methods were developed in order to separate the stem effect. They consist in the simultaneous use of a second passive fiber for stem effect subtraction, ${ }^{11}$ in the temporal discrimination between scintillation and Cerenkov light, ${ }^{12}$ or in the use of a rigid air core guide. ${ }^{13}$ Chromatic signal separation making use of interference or dichroic filters was proposed for plastic scintillators. ${ }^{14,15}$ These methods can enable the suppression of the Cerenkov light provided that an elaborate calibration procedure is followed. ${ }^{16,17}$

It is clear that the attractiveness of RL fiber dosimetry would be greatly enhanced by employing a RL signal free from any spectral superposition with spurious ones so to realize a simple and practical sensor, and avoid any kind of subtraction or correction algorithm. In this respect, a first attempt to shift the dosimetric signal in a different spectral position was made by doping silica with $\mathrm{Eu}^{3+}$ and exploiting the sharp emission line due to the ${ }^{5} \mathrm{D}_{0}-{ }^{7} \mathrm{~F}_{2}$ transition occurring in the red region at $620 \mathrm{~nm} .{ }^{18}$ However, a background due to stem effect is observable in the red region as well, and a correction procedure is still needed for truly precise evaluations. ${ }^{19,20}$ As a straightforward resolution of the problem, we here show the advantage to work in the near infrared. Specifically, we present the results related to fibers doped with ytterbium for which the RL signal is due to the ${ }^{2} \mathrm{~F}_{5 / 2}{ }^{2} \mathrm{~F}_{7 / 2}$ transition displaying a sharp emission line at about $975 \mathrm{~nm}^{21}$ We consider the effectiveness of the system 
by analyzing its sensitivity, linearity, and reproducibility under irradiation with photon fields above Cerenkov energy threshold.

Optical fiber based dosimeters were obtained by fusionsplicing (by Starlite srl, Italy) $10 \mathrm{~mm}$ long pieces of $\sim 200 \mu \mathrm{m}$ in diameter, Yb-doped fibers to commercial optical fibers. Silica glass cylinders were produced with an $\mathrm{Yb}$ molar ppm concentration of about $400 \mathrm{ppm}$ [mol. ppm $=$ (moles of RE/ (moles of $\mathrm{RE}+$ moles of $\left.\mathrm{SiO}_{2}\right) \times 10^{6}$ ] using Tetraethyl orthosilicate (TEOS, Aldrich, 99.999\%) and $\mathrm{Yb}\left(\mathrm{NO}_{3}\right)_{3} \cdot 6 \mathrm{H}_{2} \mathrm{O}$ (Aldrich, 99.99\%), as precursors. Following hydrolysis and gelation procedure in a suitable sealed cylindrical container, the resulting gel samples were aged for few days and, subsequently, small holes were produced in the container covers in order to induce slow drying of the alcogel. Drying of the alcogels was reached in about $2-3$ weeks at $35^{\circ} \mathrm{C}$, yielding crackfree cylindrical xerogels. Densification of xerogels to glasses was carried out through a sintering procedure up to $1250^{\circ} \mathrm{C}$ with a suitable thermal procedure and gases. In order to confirm the $\mathrm{Yb}$ doping level in the final glass inductively coupled plasma mass spectrometry (Perkin Elmer Elan DRC-e) with laser ablation sampler (New Wave UP213) analyses were performed using a NIST 610 glass as standard.

$\mathrm{Yb}$ doped fibers were obtained by drawing a few centimeters long glass cylinder produced by sol gel method without adding any cladding during the process. In the active portion of this sensor, the light guide is due to the interface with air.

As passive, signal carrying, fibers we used multimode commercial fibers with numerical aperture equal to 0.48 (Polymicro Technologies, USA), showing a large core diameter $(\sim 200 \mu \mathrm{m})$, surrounded by a polymeric cladding, reaching a total diameter of nominally $225 \mu \mathrm{m}$. RL measurements were performed by coupling the fiber dosimeters to a thermoelectric cooled back-thinned Charge-Coupled Device (CCD) array spectrometer (PrimeTM X, B\&WTec Inc., USA).

In order to assess the influence of the stem effect on the system response, the Yb-doped silica fiber, whose RL sensitivity had been previously stabilized as explained hereinafter, was exposed to ionizing radiation together with increasing portions of passive fiber within the radiation field. Irradiations were performed with $30 \times 30 \mathrm{~cm}^{2}$ photon fields produced by a medical linear accelerator (Clinac 2100, Varian Medical Systems) operating at $6 \mathrm{MV}$. The fiber was placed on the top flat surface of a polymethyl methacrylate (PMMA) phantom with the doped portion at the centre of the radiation field and the fiber axis orthogonal to the beam direction. A build-up bolus was positioned over the doped portion to achieve electronic equilibrium. Longer portions of passive fiber, from approximately $19 \mathrm{~cm}$ up to $174 \mathrm{~cm}$ were progressively rolled within the radiation field. We note that passive fibers portions of the order of $20 \mathrm{~cm}$ or less are the only ones realistically exposed to radiation in a medical therapy field, while longer portions were included in the experiment in order to really demonstrate the ability of the system to exclude the stem effect contribution even in extreme conditions. The resulting RL spectra are shown in Figure 1(A). The RL emission of the Yb-doped fiber was characterized by the expected narrow peak at approximately

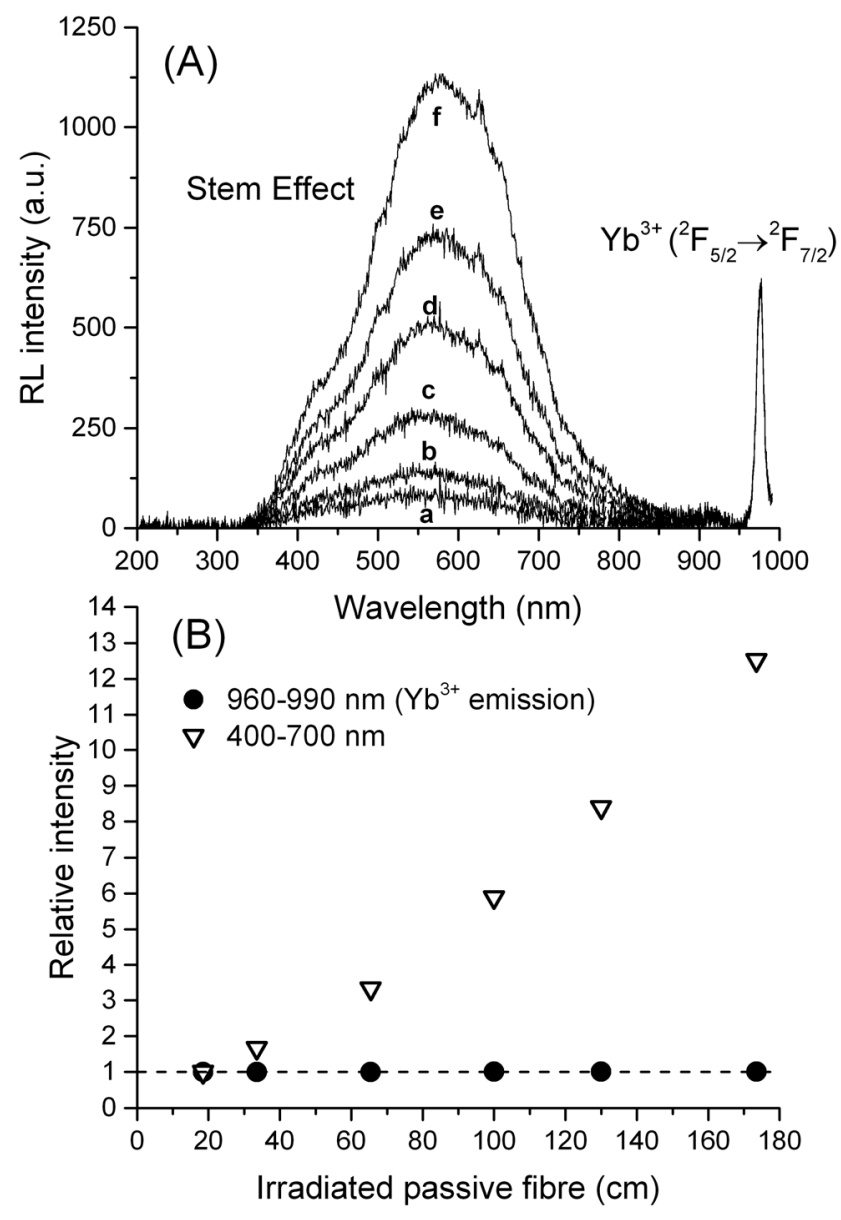

FIG. 1. (A) RL spectra of Yb-doped fiber irradiated with $6 \mathrm{MV}$ photons, together with increasing portions of passive fiber in the radiation field (a: $19 \mathrm{~cm}$; b: $34 \mathrm{~cm}$; c: $66 \mathrm{~cm}$; d: $100 \mathrm{~cm}$; e: $130 \mathrm{~cm}$; $: 174 \mathrm{~cm}$ ). (B) Relative intensities of the $\mathrm{Yb}^{3+} \mathrm{RL}$ signal and of the stem effect contribution versus the length of passive fiber exposed to ionizing radiation.

$975 \mathrm{~nm}$ of $\mathrm{Yb}^{3+}$. In addition, a strong spurious luminescence signal, namely, the stem effect, occurring at lower wavelengths was observed.

Although a direct and quantitative comparison between the intensities of the $\mathrm{Yb}^{3+} \mathrm{RL}$ signal and the stem effect contribution cannot be performed (the spectra of Fig. 1(A) being not corrected for the spectral response of the detection system) the different dependence of these two signals on the portion of passive fiber within the radiation field can be clearly observed. This aspect is well depicted in Figure 1(B), where the relative intensities of the stem effect contribution and of the $\mathrm{Yb}^{3+}$ luminescence signal were plotted versus the length of passive fiber exposed to ionizing radiation. The stem effect was evaluated by integrating the RL spectrum in the $400-700 \mathrm{~nm}$ range. In fact, as shown in Fig. 1(A), the expected spurious emission extending below $400 \mathrm{~nm}$ was not detected due to the optical fiber attenuation and the low CCD sensitivity in this spectral region. The RL signal of the doped fiber was evaluated by integrating the main emission of $\mathrm{Yb}^{3+}$ in the $960-990 \mathrm{~nm}$ spectral interval. While the stem effect critically increased with increasing the irradiated portion of passive fiber, the intensity of the $\mathrm{Yb}^{3+} \mathrm{RL}$ signal remained constant.

In order to achieve a stable $\mathrm{Yb}^{3+} \mathrm{RL}$ response, a preirradiation of the doped fiber proved to be required. Indeed, 
as for many other scintillation materials, ${ }^{21-24}$ the RL sensitivity of the $\mathrm{Yb}$-doped silica fiber was observed to increase as a consequence of a prolonged exposition to ionizing radiation.

This aspect is shown in Figure 2(A) where the relative intensity of the $\mathrm{Yb}^{3+} \mathrm{RL}$ signal was plotted versus the cumulated gamma dose absorbed by the doped fiber during the irradiation with a ${ }^{60} \mathrm{Co}$ source, at a dose rate of $195 \mathrm{~Gy} / \mathrm{h}$. The intensity of the $\mathrm{Yb}^{3+} \mathrm{RL}$ signal increased up to a saturation value, nearly equal to six times the initial value. The saturation was reached after a cumulated dose of approximately $7 \mathrm{kGy}$.

The radiation induced increase of RL sensitivity can be explained by the presence of intrinsic defects in the silica matrix that trap the free carriers, competing with luminescent centers during the carrier recombination path. ${ }^{21-24}$ This competing process, being dependent on the concentration of empty traps with respect to that of the luminescent centers, becomes less relevant as traps are progressively populated during the irradiation, and it runs out once the competing traps are totally filled.

At the end of the irradiation, the RL sensitivity was tested with $20 \mathrm{kV}$ X-rays. A slight decrease of RL sensitivity was observed, as a consequence of detrapping phenomena involving the shallower competing traps (i.e., the traps not completely stable at room temperature). This effect is shown in Figure 2(B), where the relative intensity of the $\mathrm{Yb}^{3+} \mathrm{RL}$ signal was plotted versus post-irradiation time. A decrease of the RL sensitivity from the saturation value of approximately $3 \%$ occurred in the first $15 \mathrm{~min}$ after the prolonged irradiation. Afterwards, a slower decay rate was observed: after $10 \mathrm{~h}$ post-irradiation the RL sensitivity still remained equal to $95 \%$ of the saturation value. Such percentage of the saturation value corresponds to a cumulated dose of approximately 4 kGy (see dashed horizontal line in Figure 2). Therefore, considering the shape of the curve of the RL sensitivity increase versus cumulated dose in Fig. 2(A), no significant further changes in the dosimeter response are expected in case of routinely dose monitoring up to few Gy (e.g., typical doses used in radiation therapy applications).

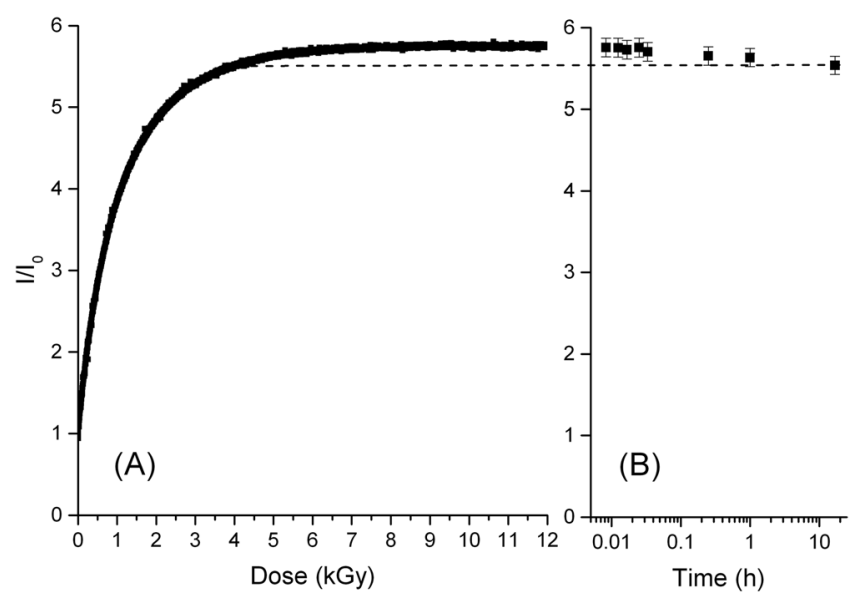

FIG. 2. Relative intensity of the $\mathrm{Yb}^{3+} \mathrm{RL}$ signal versus ${ }^{60} \mathrm{Co}$ cumulated dose (A) and versus post-irradiation time, tested with $20 \mathrm{kV} \mathrm{X}$-rays (B). The dashed horizontal line connects points with the same sensitivity in both panels.
This aspect was verified by performing sequential measurements of the $\mathrm{Yb}^{3+} \mathrm{RL}$ signal over a period of approximately 50 days, starting from $24 \mathrm{~h}$ post-irradiation to a saturation dose of $12 \mathrm{kGy}$. Each time three repeated irradiations to a dose of $2 \mathrm{~Gy}$ by using $6 \mathrm{MV}$ photon beams were carried out in order to measure the RL of the doped fiber. The optical fiber was still placed on the top surface of the PMMA phantom with the doped portion, covered by a buildup bolus, at the centre of a $10 \times 10 \mathrm{~cm}^{2}$ radiation field.

The results of this test are shown in Figure 3 where the percentage differences with respect to the mean value of the $\mathrm{Yb}^{3+} \mathrm{RL}$ signal, calculated over the entire time period, were plotted versus the elapsed time from the first measure after stabilization. Each point of Figure 3 represents the average of the three repeated measurements carried out on the same day; the error bars (1 standard deviation) are not shown as they are smaller than the symbols.

The short term repeatability, i.e., the relative standard deviation of the repeated measurements performed on the same day was evaluated to be approximately $0.5 \%$. The long term reproducibility of the system response, calculated as relative standard deviation of the entire pool of data was equal to $3 \%$. It must be pointed out that this value takes into account both possible variations in the RL of the doped fiber, and further factors, as the long term reproducibility of the spectrometer response and the slight daily deviations in the irradiation set-up positioning of the doped fiber.

In addition to a satisfying reproducibility, a linear dose rate response is required for an effective use of the Yb-doped silica fiber as RL dosimeter. This feature was verified by changing the relative distance between the doped fiber and the radiation source. In detail, the doped fiber, still placed on the top surface of the PMMA phantom and covered with the build up bolus, was orthogonally irradiated with a $10 \times 10 \mathrm{~cm}^{2} 6 \mathrm{MV}$ photon field at different distances from the $\mathrm{X}$-rays source. The dose rate interval from approximately 20 to $120 \mathrm{mGy} / \mathrm{s}$, typical of most of the radiation therapy applications, was considered. For each relative position of the fiber with respect to the source

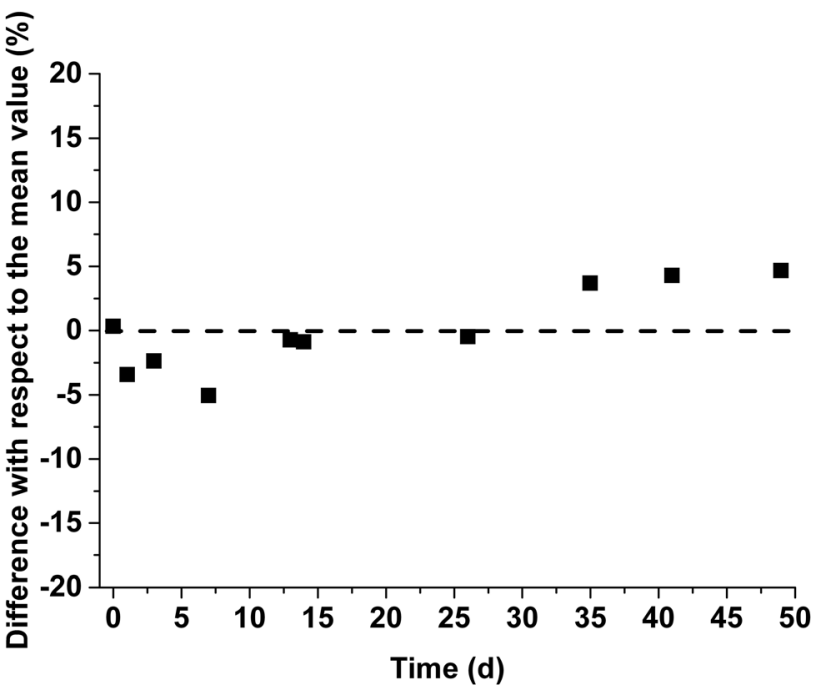

FIG. 3. Sequential measurements of the $\mathrm{Yb}^{3+} \mathrm{RL}$ signal over a period of approximately 50 days, starting from $24 \mathrm{~h}$ post-irradiation to a saturation dose of $12 \mathrm{kGy}$. 


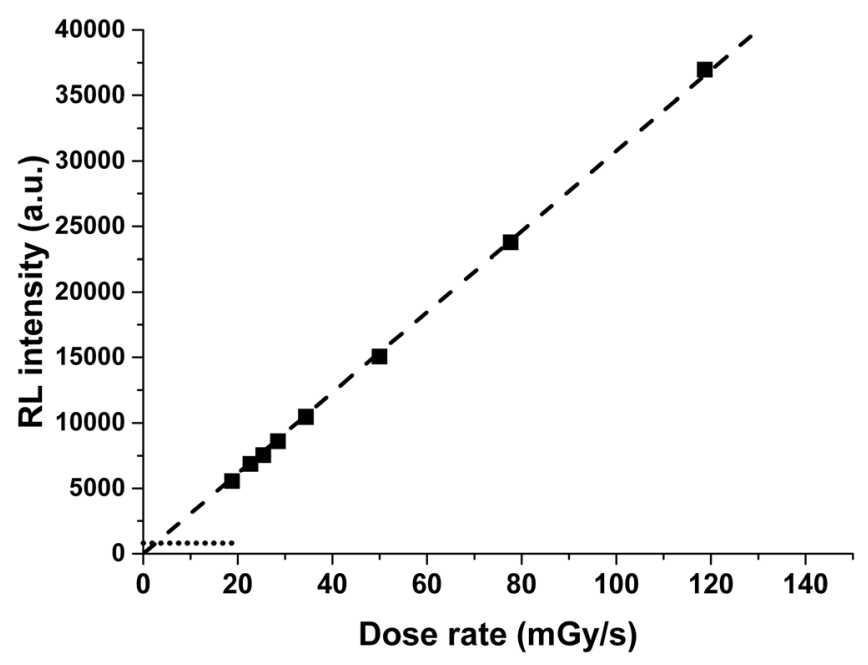

FIG. 4. Response of the $\mathrm{Yb}^{3+} \mathrm{RL}$ signal with the dose rate. The dashed line represents the linear fit of the data. The horizontal dotted line represents three times the standard deviation of the instrumental background.

(i.e., for each dose rate value), RL spectra were acquired by integrating the signal over a time period of $65 \mathrm{~s}$. The results of this test are shown in Figure 4 where the intensity of the $\mathrm{Yb}^{3+} \mathrm{RL}$ signal was plotted versus the dose rate. The RL intensity proved to be linear over the investigated interval. Assuming a linear RL response also at lower dose rates than those measured, and considering as lowest limit of detectable RL signal the value equal to three times the standard deviation of the instrumental background (horizontal dotted line in Figure 4), a minimum detectable dose rate of $2.6 \mathrm{mGy} / \mathrm{s}$ was calculated, corresponding to a minimum detectable dose of $170 \mathrm{mGy}$. This value critically depends on the optical device used to detect the $\mathrm{Yb}^{3+} \mathrm{RL}$ signal. In this work, the use of the CCD was aimed at evaluating the satisfactory spectral separation between the dosimetric and spurious signals. A significant reduction of the detection limit can be expected by coupling the Yb-doped optical fiber with a device optimized for near IR photons detection.

In conclusion, we have demonstrated that by exploiting a near infrared emitter like $\mathrm{Yb}^{3+}$ a stem effect-free dosimetric signal can be obtained with suitable linearity, reproducibility, and sensitivity properties. This paves the way to the use of Yb-doped silica fibers, widely employed in laser technology, also in ionizing radiation monitoring where an efficient stem effect removal is required, like in the presence of extended fields and high radiation energies.

The authors gratefully acknowledge the financial support of the project "Nanomed" of the Italian Ministry of
Education and Research (2013-2015). The authors are also grateful to the staff of the Laboratorio Energia Nucleare Applicata (LENA), Pavia, Italy, for their assistance during ${ }^{60}$ Co irradiations.

${ }^{1}$ R. J. Mears, L. Reckie, I. M. Jauncey, and D. N. Payne, Electron. Lett. 23, 1026 (1987).

${ }^{2}$ Y. Jeong, J. K. Sahu, D. N. Payne, and J. Nilsson, Opt. Express 12, 6088 (2004).

${ }^{3}$ J. Nilsson and D. N. Payne, Science 322, 921 (2011).

${ }^{4}$ A. Vedda, N. Chiodini, D. Di Martino, M. Fasoli, S. Keffer, A. Lauria, M. Martini, F. Moretti, G. Spinolo, M. Nikl, N. Solovieva, and G. Brambilla, Appl. Phys. Lett. 85, 6356 (2004).

${ }^{5}$ D. Di Martino, A. Vedda, G. Angella, M. Catti, E. Cazzini, N. Chiodini, F. Morazzoni, R. Scotti, and G. Spinolo, Chem. Mater. 16, 3352 (2004).

${ }^{6}$ A. Vedda, N. Chiodini, D. Di Martino, M. Fasoli, F. Morazzoni, F. Moretti, R. Scotti, G. Spinolo, A. Baraldi, R. Capelletti, M. Mazzera, and M. Nikl, Chem. Mater. 18, 6178 (2006).

${ }^{7}$ N. Caretto, N. Chiodini, F. Moretti, D. Origgi, G. Tosi, and A. Vedda, Nucl. Instrum. Methods Phys. Res., Sect. A 612, 407 (2010).

${ }^{8}$ I. Veronese, M. C. Cantone, N. Chiodini, A. Coray, M. Fasoli, A. Lomax, E. Mones, F. Moretti, and A. Vedda, Radiat. Meas. 45, 635 (2010).

${ }^{9}$ E. Mones, I. Veronese, F. Moretti, M. Fasoli, G. Loi, E. Negri, M. Brambilla, N. Chiodini, G. Brambilla, and A. Vedda, Nucl. Instrum. Methods Phys. Res., Sect. A 562, 449 (2006).

${ }^{10}$ E. Mones, I. Veronese, A. Vedda, G. Loi, M. Fasoli, F. Moretti, N. Chiodini, B. Cannillo, and M. Brambilla, Radiat. Meas. 43, 888 (2008).

${ }^{11}$ A. S. Beddar, T. R. Mackie, and F. H. Attix, Phys. Med. Biol. 37, 925 (1992).

${ }^{12}$ M. A. Clift, P. N. Johnston, and D. V. Webb, Phys. Med. Biol. 47, 1421 (2002).

${ }^{13}$ J. Lambert, Y. Yin, D. R. McKenzie, S. Law, and N. Suchiwerska, Phys. Med. Biol. 53, 3071 (2008).

${ }^{14}$ J. M. Fontbonne, G. Iltis, G. Ban, A. Battala, J. C. Vernhes, J. Tillier, N. Bellaize, C. Le Brun, B. Tamain, K. Mercier, and C. Motin, IEEE Trans. Nucl. Sci. 49, 2223 (2002).

${ }^{15}$ A. M. Frelin, J. M. Fontbonne, G. Ban, J. Colin, M. Labalme, A. Batalla, A. Isambert, A. Vela, and T. Leroux, Med. Phys. 32, 3000 (2005).

${ }^{16}$ C. E. Andersen, S. M. S. Damkjaer, G. Kertzsher, S. Greilich, and M. C. Aznar, Radiat. Meas. 46, 1090 (2011).

${ }^{17}$ P. Z. Y. Liu, N. Suchowerska, J. Lambert, P. Abolfathi, and D. R. Mckenzie, Phys. Med. Biol. 56, 5805 (2011).

${ }^{18}$ A. Baraldi, E. Buffagni, R. Capelletti, M. Mazzera, M. Fasoli, A. Lauria, F. Moretti, A. Vedda, and M. Gemmi, J. Phys. Chem. C 117, 26831 (2013).

${ }^{19}$ I. Veronese, M. C. Cantone, M. Catalano, N. Chiodini, M. Fasoli, P. Mancosu, E. Mones, F. Moretti, M. Scorsetti, and A. Vedda, J. Phys. D: Appl. Phys. 46, 015101 (2013).

${ }^{20}$ I. Veronese, M. C. Cantone, N. Chiodini, M. Fasoli, E. Mones, F. Moretti, and A. Vedda, Radiat. Meas. 56, 316 (2013).

${ }^{21}$ F. Moretti, N. Chiodini, M. Fasoli, A. Lauria, and A. Vedda, J. Non-Cryst. Solids 353, 486 (2007).

${ }^{22}$ I. Veronese, M. Fasoli, M. Martini, F. Moretti, A. Vedda, G. Loi, and E. Mones, Phys. Status Solidi C 4, 1024 (2007).

${ }^{23}$ E. Dell'Orto, M. Fasoli, G. Ren, and A. Vedda, J. Phys. Chem. C 117, 20201 (2013).

${ }^{24}$ F. Moretti, G. Patton, A. Belski, M. Fasoli, A. Vedda, M. Trevisani, M. Bettinelli, and C. Dujardin, J. Phys. Chem. C 118, 9670 (2014). 